\title{
TWO-STAGE FUSION OF THERMAL HYPERSPECTRAL AND VISIBLE RGB IMAGE BY PCA AND GUIDED FILTER
}

\author{
Wenzhi Liao ${ }^{1}$, Xin Huang ${ }^{2}$, Frieke Van Coillie ${ }^{3}$, Guy Thoonen ${ }^{4}$, Aleksandra Pižurica ${ }^{1}$, \\ Paul Scheunders ${ }^{4}$, Wilfried Philips ${ }^{1}$ \\ ${ }^{1}$ Ghent University-TELIN-IPI-iMinds, B-9000 Ghent, Belgium \\ ${ }^{2}$ The State Key Laboratory of Information Engineering in Surveying, Mapping and Remote Sensing, \\ Wuhan University, 430079 Wuhan, China \\ ${ }^{3}$ FORSIT, Department of Forest and Water Management, Gent University, B-9000 Ghent, Belgium \\ ${ }^{4}$ iMinds-Vision Lab, University of Antwerp, B-2610 Antwerp, Belgium
}

\begin{abstract}
Nowadays, advanced technology in remote sensing allows us to get multi-sensor and multi-resolution data from the same region. Fusion of these data sources for classification remains challenging problems. In this paper, we propose a novel algorithm for hyperspectral (HS) image pansharpening with twostage guided filtering in PCA (principal component analysis) domain. In the first stage, we first downsample the highresolution RGB image to the same spatial resolution of original low-resolution HS image, and use guided filter to transfer the image details (e.g. edge) of the downsampled RGB image to the original HS image in the PCA domain. In the second stage, we perform upsampling on the resulting HS image from the first stage by using original high-resolution RGB image and guided filter in PCA domain. This yields a clear improvement over an older approach with one stage guided filtering in PCA domain. Experimental results on fusion of a low spatial-resolution Thermal Infrared HS image and a high spatial-resolution visible RGB image from the 2014 IEEE GRSS Data Fusion Contest, are very encouraging.
\end{abstract}

Index Terms - Hyperspectral images, pansharpening, principal component analysis, guided filter

\section{INTRODUCTION}

Recent advances in the sensors technology of remote sensing (RS) have led to an increased availability of acquiring multi-sensor and multi-resolution data from the same area. In particular, hyperspectral (HS) images provide a detailed description of the spectral signatures of ground covers but with low spatial resolution, whereas visible RGB images with high resolution give detailed spatial information the same surveyed area.

This work was supported by the SBO-IWT project Chameleon: Domainspecific Hyperspectral Imaging Systems for Relevant Industrial Applications, and FWO project G037115N: Data fusion for image analysis in remote sensing.
Many techniques have been developed for fusion of multisensor and multi-resolution RS imagery [1, 2, 3, 4]. To superresolve the low spatial resolution HS/multispectral (MS) to the same spatial size of high resolution $\mathrm{RGB} /$ panchromatic (PAN) image, some of these approaches employ the so-called component substitution methods [1] or their generalization [2]. Others model PAN image as a linear combination of the ideal MS bands, and restore an ideal high-resolution MS image by utilizing different regularization [3]. Recently, Licciardi et al. [4] combined non-linear PCA (NLPCA) and Indusion to enhance the spatial resolution of the HS image by fusing a PAN image. Their method first applied NLPCA to project the original HS data into a lower space, then enhanced the derived nonlinear PCs by the Indusion process; finally, got the high spatial resolution HS data by inversing NLPCA. However, these approaches either suffer from spectral distortions or from high cost on computational time to estimate a good solution. An older version of our hyperspectral image pansharpening method using guided filter [8] in PCA domain (GFPCA) [5] won the "Best Paper Challenge" award of 2014 IEEE Data Fusion Contest, by fusing a low resolution (LR) Thermal Infrared HS image and a high spatial resolution visible RGB image of the same scene. However, the spatial information of high resolution (HR) RGB image was not well exploited in GFPCA [5].

In this paper, we propose a novel framework for hyperspectral image pansharpening using two-stage guided filter in PCA domain. As we know, the upsampling is the inverse problem of downsampling. This yields the condition that an upsampled image should, when downsampled, give the initial image back [6]. Therefore, the proposed method first downsamples the HR RGB image to the same spatial resolution of LR HS image, and uses guided filter [8] to transfer the image details (e.g. edge) of the downsampled RGB image to the original LR HS image in the PCA domain. In the second stage, we perform upsampling on the resulting HS image from the first stage by using original high-resolution RGB image 


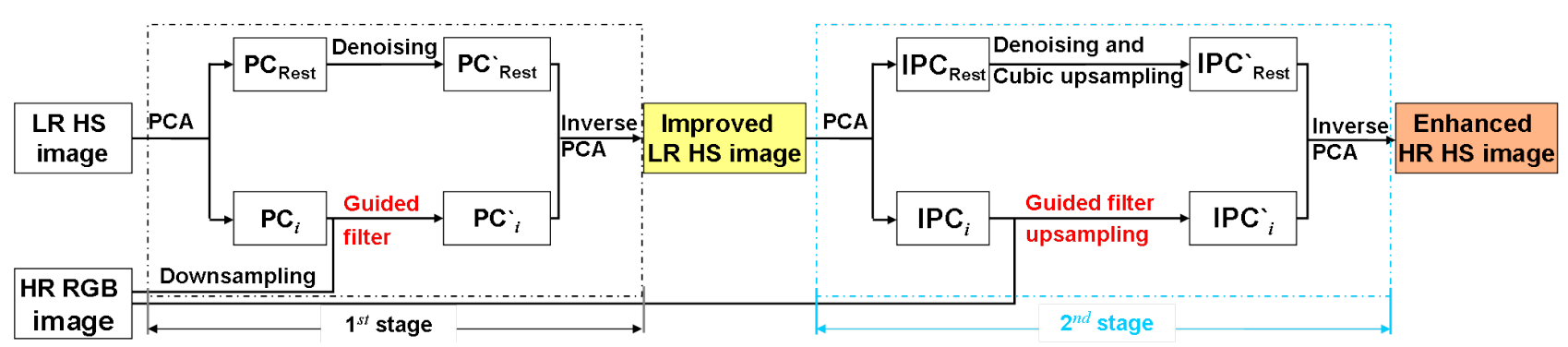

Fig. 1: Proposed framework for hyperspectral image pansharpening using two-stage guided filter in PCA domain.

and guided filter in the PCA domain. Experimental results demonstrate the proposed two-stage pansharpening method can not only preserve the original spectral information and spatial details from RGB image, but also enable better performances on classification. The remainder of the paper is organized as follows. The proposed hyperspectral pansharpening method is detailed in Section 2. Section 3 presents the experimental results. Finally, conclusions are drawn in Section 4.

\section{PROPOSED METHOD}

One of the main challenges of fusion a low spatial HS and a high resolution RGB to get a high spatial resolution HS, is not easy to make a balance on spectral and spatial preservations. Recently, the guided filter [8] has been widely used in many applications (e.g. edge-aware smoothing, detail enhancement and etc.), as its efficient and strong abilities to transfer the structures of the guidance image to the filtering output. Its application to HS data can be found in [9], where the guided filter [8] was applied to transfer the structures of the principal components of the HS image to the initial classification maps.

We developed in this section a two-stage pansharpening method for HS image. Fig. 1 shows our proposed framework. To fully exploit the spatial structures of HR RGB image and better co-register multi-sensor data, in the first stage, we first downsample the HR RGB image to the same spatial resolution of LR HS image, and use PCA to decorrelate the original LR HS images and separate the information content from the noise. The first $k$ PCA channels contain most of the total energy of a HS image (i.e. most information of the HS image), and the remaining $B-k$ PCA channels (where $B$ is the number of spectral bands of HSI and $B \gg k$ ) mainly contain noise. We use guided filter [8] to transfer the image details (e.g. edge) of the downsampled RGB image to the first $k$ PCA channels only. We remove the noise (without guided filtering) in the remaining PCA channels using a soft-thresholding scheme. The improved LR HS image can be obtained by inverse PCA. In the second stage, we use the guided filter [8] in PCA domain to transfer the image details from the original HR RGB to the first few PCs of the improved LR HS image (we get from the first stage), same as we did in [5].

Let $P C_{i}$ denote the first $i(i \leq k)$ PC of the original LR
HS image, $I_{R G B}^{\prime}$ the downsampled (to the same spatial resolution of the original LR HS image) RGB image by cubic interpolation. The filtering output $P C_{i}^{\prime}$ can be represented as a linear transform of guided image $I_{R G B}^{\prime}$ in a local window $\omega$ of size $(2 r+1) \times(2 r+1)$ as follows:

$$
P C_{i}^{\prime}=a_{j} I_{R G B}^{\prime}+b_{j}, \forall i \in \omega_{j}
$$

The above model ensures that the output $P C_{i}^{\prime}$ has an edge only if the guided image $I_{R G B}^{\prime}$ has an edge, as $\nabla P C^{\prime}=$ $a \nabla I_{R G B}^{\prime}$. The following cost function was used to find the coefficients $a_{j}$ and $b_{j}$ :

$$
\left.E\left(a_{j}, b_{j}\right)=\Sigma_{i \in \omega_{j}}\left(a_{j} I_{R G B}^{\prime}+b_{j}-P C_{i}\right)^{2}+\epsilon a_{j}^{2}\right)
$$

where $\epsilon$ is a regularization parameter determining the degree of the blurring for the guided filter. For more details about guided filter, we refer the readers to [8]. In the cost function, the $P C_{i}^{\prime}=a_{j} I_{R G B}^{\prime}+b_{j}$ should be as close as much to the $P C_{i}$, which can make sure the preservation of the original spectral information. The remaining $B-k$ PCA channels (where $B$ is the number of spectral bands of HSI and $B \gg k$ ) mainly contain noise. If guided filtering is performed on these noisy and high-dimensional $B-k$ PCs, then it will amplify the noise of the data cube and cause high computational cost in processing the data, which is undesirable. Therefore, we remove the noise (and without guided filter) in the remaining PCA channels using a soft-thresholding scheme. After the first stage, we can see from Fig. 2 that the improved LR HS image become sharper, and contain less noise compared with the original LR HS image. Moreover, the first stage ensures better co-registration of multi-sensor data for the following spatial enhancement.

In the second stage, we perform upsampling on the improved LR HS image from the first stage by combining the original HR RGB image and guided filter [8] in PCA domain, same as we did in [5]. For more details about our previous one stage hyperspectral pansharpening method by guided filter in PCA domain, the readers can find relevant information in [5, 7]. Fig. 2] shows the effectiveness of our proposed two-stage hyperspectral image pansharpening method in spectral and spatial preservations. From a visual analysis, the fused image produced by the proposed approach appears to be sharper than our previous method [5], where only one stage of guided filtering was performed in PCA domain. 

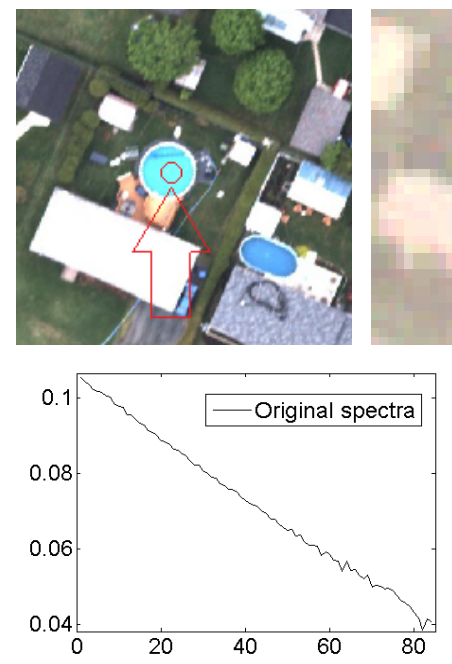
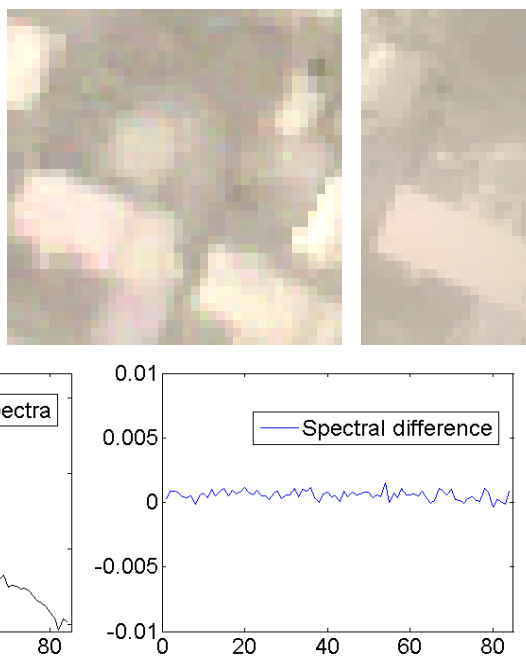
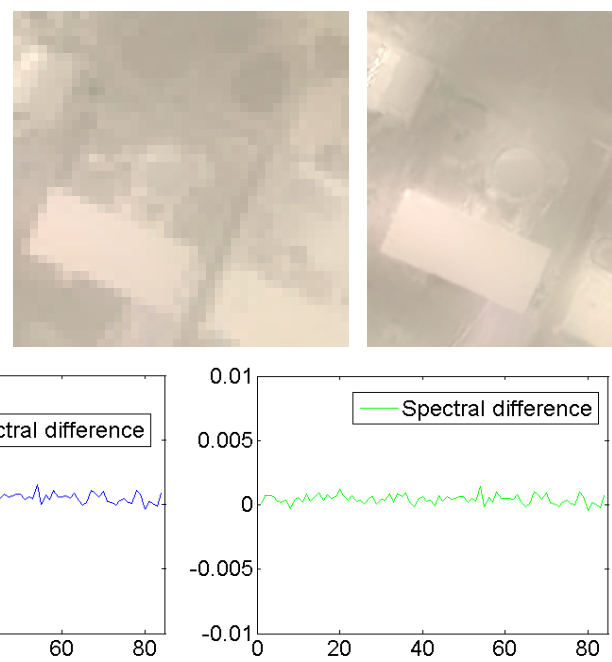
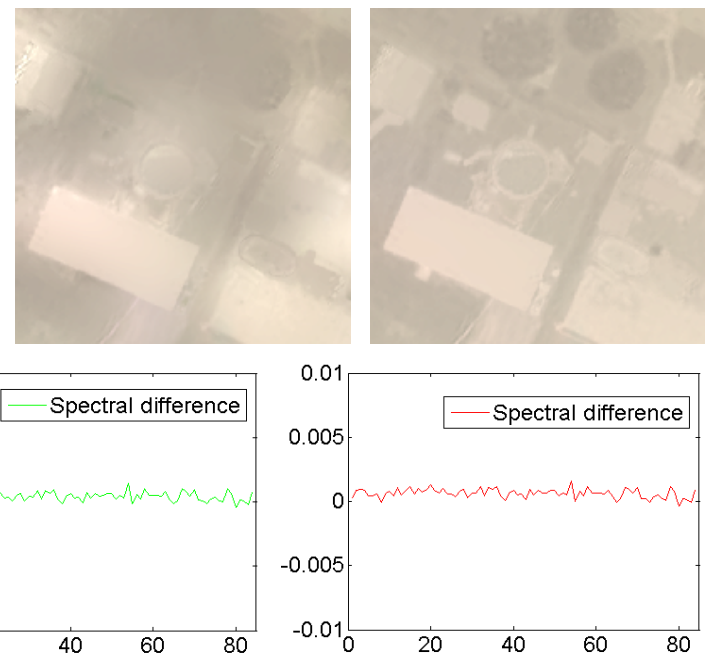

Fig. 2: Part of enhanced TI HS image. The top row shows HR RGB image, three bands composition by original LR HS image, improved LR HS image (in the first stage), enhanced images by GFPCA [5] and by our proposed method; the second row shows the original spectra and its difference with improved LR HS image, GFPCA [5] and our proposed method.

Table 1: Average classification accuracies and consumed time (hour) obtained according to the described scheme.

\begin{tabular}{|c|c|c|c|c|c|}
\hline & Cub & RGB & PCA & GFPCA & Proposed \\
\hline Feat. & 84 & 84 & 84 & 84 & 84 \\
\hline OA (\%) & 52.6 & 77.2 & 59.9 & 79.0 & $\mathbf{8 4 . 3}$ \\
AA (\%) & 35.1 & 77.9 & 54.6 & 66.8 & $\mathbf{7 8 . 5}$ \\
$\kappa(\%)$ & 34.7 & 67.5 & 46.3 & 68.7 & $\mathbf{7 6 . 7}$ \\
\hline Time (h) & 3.81 & $\mathbf{0 . 2 8}$ & 2.82 & 1.17 & 1.23 \\
\hline
\end{tabular}

Moreover, the spectral preservations of our proposed method and [5] are similar, and spectrally consistent with respect to the original HS image. With only one stage fusion, GFPCA [5] mistook some big bright objects in the original LR HS as a object in the enhanced HS, leading to poor preservation of spatial information.

\section{EXPERIMENTAL RESULTS}

Experiments are done on a thermal infrared hyperspectral data and a visual RGB image which were acquired by Telops Inc. on May 2013 over an urban area near Thetford Mines in Québec, Canada. The TI HS image has 84 spectral bands that covers the wavelengths between 7.8 to $11.5 \mu \mathrm{m}$ with approximately 1- $m$ spatial resolution. The visible RGB image is a series of color images acquired during separate flight-lines with approximately $20-\mathrm{cm}$ spatial resolution. The whole scene of both data contains 7 classes, but with different spatial size of which the TI HS consists of $874 \times 751$ pixels while RGB of $4386 \times 3769$. Fig. 3 shows an RGB composition with the labeled classes highlighted, for details, see [10].

The SVM classifier with radial basis function (RBF) kernels is applied in our experiments. We apply a gridsearch on the two parameters $C$ and $\gamma$ of SVM using 5fold cross-validation to find the best $C$ within the given set $\left\{10^{-1}, 10^{0}, 10^{1}, 10^{2}, 10^{3}\right\}$ and the best $\gamma$ within the given set $\left\{10^{-3}, 10^{-2}, 10^{-1}, 10^{0}, 10^{1}\right\}$. We compare our proposed hyperspectral pansharpening method with the schemes of (1) Simply enlarging the original HS image by cubic interpolation (Cub); (2) Only using the visible RGB image (RGB); (3) PCA component substitution method (PCA), similar as [1]; (4) Our previous method using guided filter in PCA domain (GFPCA) [5]. For quantitative comparisons, we randomly select 1000 samples per class from training set for training, the results are averaged over five runs. The classification results are quantitatively evaluated by measuring the Overall Accuracy (OA), the Average Accuracy (AA) and the Kappa coefficient $(\kappa)$ on the test data. The experiments were carried out on 64-b, $3.40 \mathrm{GHz}$ Intel i7-4930K (1 core) CPU computer with 64 GB memory, the consumed time includes image fusion, feature fusion and classification. Table 1 shows the accuracies and consumed time (hours) obtained from the experiments, Fig. 3 shows the best result of each method.

It is obvious that using single data source is not enough for reliable classification. By using only the spatial information from HR RGB image, we produce better results than simply upsampling the original HS image by cubic interpolation. However, the remote sensing data from urban area was a mix between man-made structures and natural materials, different objects may share similar spatial information. For example, the spatial information of 'Red roof' and 'Grey roof' or 'Bare soil' is similar, objects from 'Red roof' are misclassified as soil by only using RGB image. Image fusion by Cub cannot preserve the spatial information, leading to spatial distortions in the final classification map; whereas the PCA component substitution suffers from spectral distortions. By using the guided filter, the GFPCA performs better on both spectral and spatial preservations, and this is reflected in classification accuracy $20 \%$ higher than Cub and PCA, respectively. 


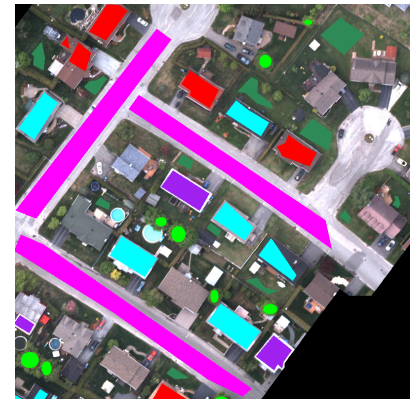

(a) Ground truth

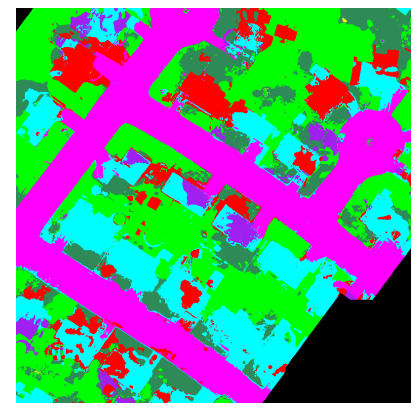

(c) GFPCA

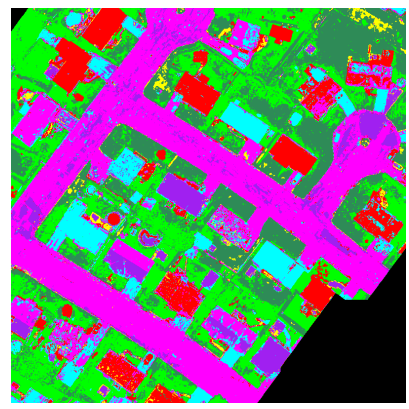

(b) RGB

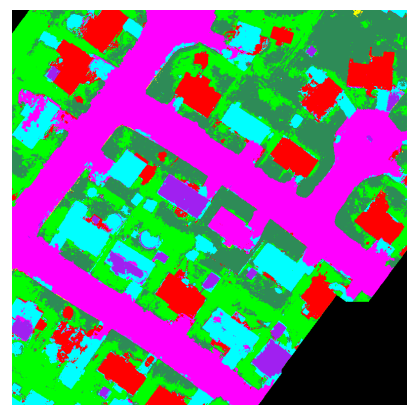

(d) Proposed
Road Trees Red roof Grey roof Concrete roof Vegetation Bare soil

Fig. 3: Part of classification maps obtained by each method.

By using the proposed two-stage pansharpening method, we have more than 5\% improvements in accuracies over the others fusion schemes. Although consuming a little bit more time than one stage pansharpening method [5], our proposed two-stage hyperspectral image pansharpening method benefits better preservations of image edges in its classification map.

\section{CONCLUSION}

The contribution of this paper is a methodology to fuse a low spatial resolution HS image and a high resolution RGB image in the classification task. Some existing image fusion methods suffer either spectral distortions or spatial distortions.By using two-stage guided filter in PCA domain, our proposed image fusion method can preserve both spectral (from original low-resolution HS image) and spatial (from high-resolution visible RGB image) information, and enable better performances on classification. Experimental results on the classification of the real TI HS and RGB images show the efficiency of the proposed method.

\section{Acknowledgment}

The authors would like to thank Telops Inc. (Québec, Canada) for acquiring and providing the data used in this study, the IEEE GRSS Image Analysis and Data Fusion Technical Com- mittee and Dr. Michal Shimoni (Signal and Image Centre, Royal Military Academy, Belgium) for organizing the 2014 Data Fusion Contest, the Centre de Recherche Public Gabriel Lippmann (CRPGL, Luxembourg) and Dr. Martin Schlerf (CRPGL) for their contribution of the Hyper-Cam LWIR sensor, and Dr. Michaela De Martino (University of Genoa, Italy) for her contribution to data preparation.

\section{REFERENCES}

[1] V. Shah, N. Younan, and R. King, "An efficient pansharpening method via a combined adaptive PCA approach and contourlets," IEEE Trans. Geosci. Remote Sens., vol. 46, no. 5, pp. 1323-1335, May 2008.

[2] B. Aiazzi, S. Baronti, and M. Selva, "Improving component substitution pansharpening through multivariate regression of MS + Pan data," IEEE Trans. Geosci. Remote Sens., vol. 45, no. 10, pp. 3230-3239, Oct. 2007.

[3] S. Li, H. Yin, and L. Fang, "Remote sensing image fusion via sparse representations over learned dictionaries," IEEE Trans. Geosci. Remote Sens., vol. 51, no. 9, pp. 4779-4789, Sep. 2013.

[4] G. Licciardi, M. M. Khan, J. Chanussot, A. Montanvert, L. Condat and C. Jutten: "Fusion of hyperspectral and panchromatic images using multiresolution analysis and nonlinear PCA band reduction," EURASIP J. Adv. Sig. Proc., vol. 2012 no. 207, pp. 1-17, 2012.

[5] W. Liao, X. Huang, F. Coillie, S. Gautama, A. Pizurica, W. Philips, H. Liu, T. Zhu, M. Shimoni, G. Moser, and D. Tuia, "Processing of Multiresolution Thermal Hyperspectral and Digital Color Data: Outcome of the 2014 IEEE GRSS Data Fusion Contest," IEEE J. Select. Top. Appl. Earth Observ. Remote Sensing, In Press.

[6] M. M. Khan, J. Chanussot, L. Condat, and A. Montanvert, "Indusion: Fusion of multispectral and panchromatic images using the induction scaling technique," IEEE Geoscience and Remote Sensing Letters, vol. 5, no.1, pp. 98102, Jan. 2008.

[7] L. Loncan, L. B. Almeida, J. Bioucas Dias, X. Briottet, J. Chanussot, N. Dobigeon, S. Fabre, W. Liao, G. Licciardi, M. Simoes, J-Y. Tourneret, M. Veganzones, G. Vivone,Q. Wei, N. Yokoya, "Introducing Hyperspectral Pansharpening," IEEE Geoscience and Remote Sensing Magazine, Accepted.

[8] K. He, J., and X. Tang, “Guided Image Filtering," IEEE Transactions on Pattern Analysis and Machine Intelligence, vol. 35, no. 6, pp. 1397-1409, 2013

[9] X. Kang, S. Li, J. A. Benediktsson, "Spectral-spatial hyperspectral image classification with edge-preserving filtering," IEEE Transactions on Geoscience and Remote Sensing. vol. 52, no. 5, pp. 2666-2677, May, 2014.

[10] 2014 IEEE GRSS Data Fusion Contest, Online: http://www.grss-ieee.org/community/technicalcommittees/data-fusion-contest/. 